\title{
A Study of Microbiological Profile in patients with Acute Pyelone- phritis in a Tertiary Care Hospital, Nandyal
}

\author{
Pogula Nagarjuna Reddy
}

Assistant professor, Department of General Medicine, Santhiram Medical College and General Hospital, Nandyal.

Corresponding author mail id: arjun.pogula@gmail.com DOI: 10.47799/pimr.0803.14

(C) 2020-21 Prathima Institue of Medical Sciences
Amikacin resistance was found $17.86 \%$ and $3.6 \%$ of the isolates. Nitrofurantoin resistance was found in 2 (7.1\%) of isolates.

Conclusion: The mean age of the study population was 57.480 \pm 11.2072 years in the study population, and there was a slight female preponderance in the occurrence of APN.The majority of participants (78\%) were distributed ina 50 to 69 -year age group.Diabetes, hypertension, ureteric calculus, and past history of UTI were the common risk factors identified in the study population.In. More than half (58\%) of the study population had a culture-positiveinfection.TheThe most common organism isolated in the study population was E.Coli, responsible for $44 \%$ of the cases. None of them were resistant to Cefoperazone plus Sulbactum or Piperacillin plus tazobactam. The other antimicrobials, which have not shown any resistance, were Meropenem and Colistin.The highest proportion of resistance was reported for Ampicillin in $82.1 \%$, followed by Levofloxacin in $67.9 \%$ of isolates. ICU admission rate was higher in culture-positive cases (63.6\%), as compared to culture-negative cases (36.4\%)None of the study subjects had hospital mortality.

\section{INTRODUCTION}

Acute pyelonephritis is a bacterial infection that affects the renal parenchyma that can be life-threatening and often leads to renal scarring. It usually ascended from the lower urinary tract, and also reaches the kidney via the bloodstream. Early diagnosis and management of acute pyelonephritis have a better impact on patient outcomes. Acute pyelonephritis is one of the severe conditions with high mortality and morbidity. It represents the most severe form of urinary tract infection. Acute pyelonephritis is the most common cause of communityonset bacteremia in elderly patients1.

\section{AIM AND OBJECTIVES:}

To study the microbiological profile in patients with acute pyelonephritis admitted in a tertiary care hospital.

\section{MATERIALS AND METHODS:}

A hospital-based prospective study was conducted in the Department of General Medicine, Santhiram Medical College, and General Hospital for a 2 year period after taking approval from the Hospital Ethics and Research Committee.

Sampling Technique and Sample Size: Universal Sampling Technique was used for the selection of study subjects. All the 
patients coming to the medicine department during the study period and fulfilling the inclusion criteria were taken for study after taking prior informed consent. The patients included in the study were from both ICU and wards. The final sample size came to be 50 subjects.

\section{Inclusion Criteria}

1. Age $>18$ years with written informed consent.

2. Patients presenting with features of acute pyelonephritis (fever with flank pain, positive urine culture, imaging suggestive of acute pyelonephritis).

\section{Exclusion criteria}

1 . Age $<18$ years.

2. Pregnant and lactating mothers.

3. Patients without informed written consent.

\section{Data Analysis}

All patient profiles were recorded in proforma, and findings were tabulated, SPSS24 was used for the analysis of the data.

\section{RESULTS:}

The proportion of the male and female population was $40 \%$ and $60 \%$, respectively in the study population.

Table 1: Descriptive analysis of Gender in the study population $(\mathrm{N}=50)$.

\begin{tabular}{|c|c|c|c|}
\hline \multicolumn{3}{|c|}{ SEX } \\
\hline \multirow{3}{|c|}{} & Frequency & Percent \\
\hline \multirow{3}{*}{ SEX } & MALE & 20 & 40.0 \\
\cline { 2 - 4 } & FEMALE & 30 & 60.0 \\
\cline { 2 - 4 } & Total & 50 & 100.0 \\
\hline
\end{tabular}

Among the study population, 29 (58\%) pf the subjects had a culture-positive infection.

Table 2: Descriptive analysis of culture status in the study population $(\mathrm{N}=50)$.

\begin{tabular}{|c|c|c|c|}
\hline \multicolumn{4}{|c|}{ CULTURE STATUS } \\
\hline \multicolumn{2}{|c|}{} & Frequency & Percent \\
\hline \multirow{3}{*}{$\begin{array}{c}\text { CULTURE } \\
\text { STATUS }\end{array}$} & YES & 29 & 58.0 \\
\cline { 2 - 4 } & NO & 21 & 42.0 \\
\cline { 2 - 4 } & Total & 50 & 100.0 \\
\hline
\end{tabular}

E. coli was the most common organism isolated in culture, in 22 (44\%) of the study subjects. Klebsiella pneumoniae was isolated in 2(4\%) subjects. Candida Albicans, Citrobacter, Enterococcus Faecalis, and Pseudomonas were isolated in 1 (2\%) subject each. One subject had a dual infection with $\mathrm{E}$. coli and Candida.
Table 3: Descriptive analysis of urine culture in the study group $(\mathrm{N}=50)$

\begin{tabular}{|c|c|c|c|}
\hline \multicolumn{4}{|c|}{ URINE CULTURE ORGANISM } \\
\hline \multicolumn{1}{|c|}{$\begin{array}{c}\text { URINE } \\
\text { CULTURE } \\
\text { ORGANISM }\end{array}$} & STERILE & Frequency & Percent \\
\cline { 2 - 4 } & E.COLI & 21 & 42.0 \\
\cline { 2 - 4 } & KLEBSIELLA PNEUMONIAE & 22 & 44.0 \\
\cline { 2 - 4 } & CANDIDA ALBICANS & 1 & 4.0 \\
\cline { 2 - 4 } & CITROBACTER & 1 & 2.0 \\
\cline { 2 - 4 } & E.COLI, CANDIDA GROWTH & 1 & 2.0 \\
\cline { 2 - 4 } & ENTEROCOCCUS FAECALIS & 1 & 2.0 \\
\cline { 2 - 4 } & PSEUDOMONAS & 1 & 2.0 \\
\cline { 2 - 4 } & AERUGINOSA & 50 & 100.0 \\
\cline { 2 - 4 } & Total & 2.0 & 2.0 \\
\hline
\end{tabular}

Among the 29 culture isolates, none of them were resistant to Cefoperazone plus Sulbactum or Piperacillin plus tazobactam. The other antimicrobials, which have not shown any resistance, were Meropenem and Colistin. The highest proportion of resistance was reported for Ampicillin as $82.1 \%$ of isolates were resistant. Levofloxacin resistance was found in $67.9 \%$ of isolates. Gentamicin and Amikacin resistance was found $17.86 \%$ and $3.6 \%$ of the isolates. Nitrofurantoin resistance was found in $2(7.1 \%)$ of isolates.

Table 4: Descriptive analysis of Antibiotic sensitivity pattern in culture-positive cases ( $N=29)$

\section{ANTIBIOTIC SENSITIVITY PATTERN IN CULTURE POSITIVE CASES}

\begin{tabular}{|c|c|c|c|c|}
\hline ANTIBIOTIC & SENSITIVE & $\%$ & RESISTANT & $\%$ \\
\hline $\begin{array}{c}\text { CEFOPERAZONE + } \\
\text { SULBACTUM }\end{array}$ & 29 & 100 & 0 & 0 \\
\hline $\begin{array}{c}\text { PIPERACILLIN + } \\
\text { TAZOBACTAM }\end{array}$ & 29 & 100 & 0 & 0 \\
\hline MEROPENEM & 29 & 100 & 0 & 0 \\
\hline COLISTIN & 29 & 100 & 0 & 0 \\
\hline AMIKACIN & 28 & 96.4 & 1 & 3.6 \\
\hline NITROFURANTOIN & 27 & 92.9 & 2 & 7.1 \\
\hline GENTAMICIN & 24 & 82.14 & 5 & 17.86 \\
\hline LEVOFLOXACIN & 9 & 32.1 & 19 & 67.9 \\
\hline AMPICILLIN & 5 & 17.9 & 23 & 82.1 \\
\hline TotaI & & & & \\
\hline
\end{tabular}




\section{Discussion:}

Acute pyelonephritis is an infection that affects renal parenchyma and renal pelvis. Acute pyelonephritis is considered uncomplicated if the infection is caused by a typical pathogen in an immunocompetent patient who has normal urinary tract anatomy and renal function. The condition is not generally associated with high levels of mortality, but patients can become acutely ill and experience severe pain. It can lead to sepsis, renal abscesses, and chronic pyelonephritis and also cause secondary hypertension, renal failure, and death if misdiagnosed. Acute pyelonephritis is a major complication of pregnancy that may result in significant maternal and fetal morbidity. ${ }^{2}$ The risk of APN is also increased in diabetics. Even though APN have good treatment options, developing countries like India, where there is poor access to good health services and also a high load of diabetes, make APN more complicated. 3,4

Females are prone to develop acute pyelonephritis because urinary tract infections are more common in females due to shorter urethra, hormonal changes, and close distant to the anus.

In the study of Shin et al.(2012), the proportion of females (98.3\%)was higher than males. ${ }^{5}$ In the study of Chung, V. Y.(2014) also study population consists $70.6 \%$ of females and $29.4 \%$ of males. ${ }^{6}$ In Leelavathi Venkatesh et al.( 2017), study, the most common co morbid condition was diabetes mellitus(69\%). This study concluded that diabetes mellitus patients are at high risk of developing pyelonephritis. When diagnosed early, the majority of patients respond to antibiotics. $^{7}$

In L Umesha et al. study, urine culture was positive in 48 percent of cases. Culture negativity in the study group attributed to prior usage of antibiotics in outside hospitals or due to the late collection of urine samples. This study group concluded that culture negativity groups are at lesser risk in developing complications such as renal abscess, renal failure, multi-organ dysfunction, and death. Escherichia coli is the most common pathogen in emphysematous pyelonephritis and nonemphysematous pyelonephritis groups, followed by Klebsiella pneumonia in this study group. In this study, there is a difference in antibiotic sensitivity patterns in inpatient in wards and intensive care patients though the prevalence of organisms was the same in both groups. Local antibiogram pattern is important to prevent resistances in organisms. ${ }^{8}$

In the study of Shin et al., seven hundred nineteen patients (56.8\%) had positive culture results, and 211 of them (29.3\%) also had positive blood cultures. The etiologic agents of the acute pyelonephritis in the study population, Escherichia coli was overwhelmingly the most common pathogen $(661 / 719$, $91.9 \%)$. Klebsiella pneumoniae (15/719, 2.1\%), Streptococcus species ( $7 / 719,1.0 \%)$, and Enterococcus species $(11 / 719,1.6 \%)$ were also identified. This study concluded that
Fluoroquinolone treatment of uncomplicated acute pyelonephritis caused by Fluoroquinoloneresistant Escherichia coli has lesser cure rates and prolonged hospital stay. ${ }^{5}$ In the study of Chung, V. Y. et al. the yields of blood culture were positive in $57.4 \%$ of the patients, with Escherichia coli being the commonest causative organism (38.2\%) followed by Klebsiella pneumoniae, Proteus mirabilis, and Acinetobacter species. $75 \%$ of patients in this study group had urinary obstruction. Factors attributed to mortality are old age (more than 65 years), males, renal failure, and disseminated intravascular coagulation. This study concluded there was a high prevalence of septic shock and bacteraemia in patients with severe acute pyelonephritis. ${ }^{6}$.

\section{Conclusion:}

These are the following findings from this study;

- The mean age of the study population was $57.480 \pm 11.2072$ years in the study population, and there was a slight female preponderance in the occurrence of APN.The majority of participants (78\%) were distributed in a 50 to 69-year age group.

- Diabetes, hypertension, ureteric calculus, and past history of UTI were the common risk factors identified in the study population.

- In the present study, the classical triad of pyelonephritis is present in 34 percent of patients. Fever was a predominant symptom in $84 \%$ of patients, flank pain in $78 \%$ of patients, and burning micturition in $58 \%$ of patients.

- More than half (58\%) of the study population had a culturepositive infection

- The most common radiological findings were renal calculus, ureteric calculus, hydroureteronephrosis, and bulky kidneys.

- The most common organism isolated in the study population was E.Coli, responsible for $44 \%$ of the cases.

- None of them were resistant to Cefoperazone plus Sulbactum or Piperacillin plus tazobactam. The other antimicrobials, which have not shown any resistance, were Meropenem and Colistin.

- The highest proportion of resistance was reported for Ampicillin in $82.1 \%$, followed by Levofloxacin in $67.9 \%$ of isolates.

- ICU admission rate was higher in culture-positive cases (63.6\%), as compared to culture-negative cases (36.4\%)

- None of the study subjects had hospital mortality.

\section{REFERENCES}

1. Johnson JR, Russo TA. Acute Pyelonephritis in Adults. $\mathbf{N}$ Engl J Med. 2018 Jan 4.378 (1):48-59.

2. Hill JB, Sheffield JS, Mclntire DD, Wendel Jr GD. Acute pyelonephritis in pregnancy. Obstetrics \&Gynecology. 2005; 105(1):18-23. 
3. Kumar S, Ramachandran R, Mete U, Mittal T, Dutta P, Kumar V, Rathi M, Jha V, Gupta KL, Sakhuja V, Kohli HS. Acute pyelonephritis in diabetes mellitus: Single center experience. Indian J Nephrol. 2014 Nov;24(6):367-71.

4. Kim Y, Wie SH, Chang UI, Kim J, Ki M, Cho YK, et al. Comparison of the clinical characteristics of diabetic and non-diabetic women with community-acquired acute pyelonephritis: a multicenter study. J Infect. 2014;69(3):244-51.

5. Shin J, Kim J, Wie SH, Cho YK, Lim SK, Shin SY, et al. Fluoroquinolone resistance in uncomplicated acute pyelonephritis: epidemiology and clinical impact. Microb Drug Resist. 2012; 18(2):169-75.

6. Chung, V., Tai, C., Fan, C., and Tang, C. Severe acute pyelonephritis: a review of clinical outcome and risk factors for mortality. Hong Kong Medical Journal, 2014; 20(4):285-9.

7. Venkatesh L, Hanumegowda RK. Acute pyelonephritiscorrelation of clinical parameter with radiological imaging abnormalities. Journal of Clinical and Diagnostic Research: JCDR. 2017 Jun;11(6):TC15.

8. Umesha L, Shivaprasad SM, Rajiv EN, Kumar MMS, Leelavathy V, Sreedhara CG, Niranjan MR, Acute Pyelonephritis: A Single-center Experience, Indian J Nephrol. 2018 Nov-Dec; 28(6):454-461.

How to cite this article : Nagarjuna Reddy P. A Study of Microbiological Profile in patients with Acute Pyelonephritis in a Tertiary Care Hospital, Nandyal.Perspectives in Medical Research 2020; 8 (3):61-69.

DOI: 10.47799/pimr.0803.14

Sources of Support: Nil, Conflict of interest: None declared 